\title{
Constraints on Dark Energy Models from Selected Galaxy Clusters (S-Z + X-Ray Data) and Gravitational Lensing Data
}

\author{
Alexander Bonilla Rivera *, Jairo Ernesto Castillo Hernandez \\ Facultad Tecnológica, Grupo de Investigación en Física, Matemáticas y Computación (FizMaKo), Línea de Investigación en Cosmología y Astrofísica, \\ Unversidad Distrital Francisco José de Caldas, Carrera 7 No. 40B - 53, Bogotá, Colombia \\ * Correspondence: abonillar@udistrital.edu.co
}

\begin{abstract}
The Sunyaev-Zeldovich effect (SZe) is a global distortion of Cosmic Microwave Bckground (CMB) spectrum as result of its interaction with a hot electron plasma in the intracluster medium for large gravitational virialized structures such as galaxy clusters. Furthermore, this hot gas of electrons emits X-Rays due to its fall in the gravitational potential well of the cluster. The analysis of SZe and X-Ray data, provide a method for calculating distances to galaxy clusters at any redshift (Angular diameter distance $\left(d_{A}\right)$ and gas mass fraction $\left.\left(f_{\text {gas }}\right)\right)$. On the other side, many of these galaxy clusters produce a Strong Gravitational Lens effect (SGL), which has become an useful astrophysical tool for cosmology. We use these cosmological tests, in addition to the more traditional ones (SNIa, CMB, BAO), to constraint alternative models of dark energy ( $\omega C D M, \mathrm{CPL}$, IDE, EDE) and study the history of expansion through the cosmographic parameters $(\mathrm{H}(\mathrm{z}), \mathrm{q}(\mathrm{z}), \mathrm{j}(\mathrm{z}))$. Using Akaike and Bayesian Information Criterion (AIC, BIC) we find that the $\omega C D M$ and $\Lambda C D M$ models are the most favored by the observational data. In addition, we found that at low redshift appears an peculiar behavior of slowdown of aceleration, which occurs only on dynamical dark energy models using only galaxy clusters $\left(d_{A, \text { clusters }}+f_{\text {gas }}\right)$.
\end{abstract}

Keywords: Sunyaev-Zeldovich effect, galaxy clusters, dark energy models 2016 MSC: 12th International Colloquium on Atomic Spectra and Oscillator Strengths for Astrophysical and Laboratory Plasmas

\section{Introduction}

Several authors have used the Sunyaev-Zeldovich effect (SZe), X-rays and strong gravitational lensing (SGL) data from galaxy clusters to provide independent estimations of cosmological parameters. The combination of X-rays and the SZe data lead to two useful cosmological tests: the angular diameter distance $d_{A}$ [19] and the gas mass fraction $f_{\text {gas }}$ of the galaxy clusters [13]. For instance, [19] combined the SZe and X-rays observations from 25 galaxy clusters to constrain their three-dimensional shape. Thus, they can infer the cluster $d_{A}$ which can be used to estimate cosmological parameters. [13] calculated the $d_{A}$ of a 38 galaxy clusters with redshifts $0.14 \leq z \leq 0.89$ to measure the Hubble parameter. Their estimation of $H_{0}$ is in agreement with other cosmological probes. [4], using SZe and X-rays observations, determine $f_{\text {gas }}$ of 26 galaxy clusters with redshifts $0.07 \leq z \leq 0.9$ to constrain the density parameter and the EoS of DE. Additionally, the SGL observations can be used as an additional method to probe the nature of dark matter and dark energy. [10] use a sample of 80 strong gravitational lensing (SGL) galactic systems from Sloan Lens ACS (SLACS), Lens Structure and Dynamics (LSD) surveys and galaxy clusters to fit the

Email addresses: abonillar@udistrital .edu.co (Alexander Bonilla Rivera*), jcastillo@udistrital. edu. co (Jairo Ernesto Castillo Hernandez) parameters of popular cosmological models. They found their parameter estimation is consistent with those obtained from the classical cosmological tests. Thus, we use these tools from galaxy clusters as independent complementary probes to put constraints on cosmological models.

\section{Cosmological constraints using galaxy clusters}

The galaxy clusters are the biggest gravitational structures in the Universe. They are in the transition between the linear and non-linear regimes of the structure formation. Gravitational lensing of background sources produced by these systems are used to infer the shape of matter distributions in the Universe. Nevertheless, some lensing results such as high Navarro-FrenkWhite concentration parameters and the predictions of the Einstein radii distributions are in tension with the standard $\Lambda \mathrm{CDM}$ model [9]. Therefore, the study of galaxy clusters is very important for cosmology because they offer information that can be used to develop cosmological tests that help to distinguish between different models of DM and DE. In the following, we describe three differents data from galaxy clusters that can be used to develop these tests: the SZe, X-ray and SGL.

\subsection{Angular diameter distance using S-Z/X-Ray method}

The SZe [33, 35] is a small distortion in the CMB spectrum due to the inverse Compton scattering of the CMB photons 
when they pass through the hot gas of galaxy clusters. This small fluctuation in the CMB temperature scales as $\Delta T_{S Z} \propto$ $T_{C M B} \int n_{e} T_{e} d l$, where $T_{C M B}$ is the temperature of CMB, $n_{e}$ and $T_{e}$ are the electron number density and temperature of the hot gas, respectively, and the integration is along the line of sight. The X-ray emission scales as $S_{x} \propto \int n_{e}^{2} \Lambda_{e e} d l /(1+z)^{4}$, where $\Lambda_{e e}$ is the X-ray cooling function and $z$ is the cluster redshift[13, 19]. With these two relations the cluster angular diameter distance can be infered as

$$
d_{A, \text { cluster }}(z) \propto \frac{\Delta T_{S Z}^{2}}{T_{C M B}^{2} S_{x}(1+z)^{4}},
$$

which is an experimental quantity that depends basically on the physical properties of the cluster and its geometry [19]. This method for measuring distances is completely independent of other techniques and it can be used to measure distances at high redshifts directly. We use the estimations of the $d_{A}$ from the sample of 25 galaxy clusters of [19] to constrain cosmological models. We use the 25 measurements of angular diameter distances $\left(d_{A, c}\right)$ from galaxy clusters as obtained through SZ/XRay method by De Filippis et al. (2005) [19]. In our analysis we use a maximum likelihood determined by a $\chi^{2}$ statistics, give by

$$
\chi_{d i s}^{2}\left(z_{i}, \Theta\right)=\sum_{i=1}^{25} \frac{\left(d_{A}\left(z_{i}, \Theta\right)-d_{A, c}\left(z_{i}, \Theta\right)\right)^{2}}{\sigma_{d_{c}}^{2}},
$$

where $d_{A, c}\left(z_{i}, \Theta\right)$ is the observational angular diameter distance, whose data are given by [19] and $\sigma_{d_{c}}^{2}$ is the contribution of the statistical errors. $d_{A}\left(z_{i}, \Theta\right)$ is the diametral angular distance which depends on the model of the universe.

\subsection{The gas mass fraction $f_{\text {gas }}$}

Other independent cosmological technique is to derive $d_{A}$ using the gas mass fraction data from galaxy clusters. In order to use $f_{\text {gas }}$ as cosmological test we need to assume that there is a proportion between the baryonic fraction of the galaxy clusters and the global fraction of baryonic matter and dark matter of the Universe. Moreover, it is necessary to assume that the baryonic fraction from clusters does not depend on the redshift [? ]. This assumption is valid if one considers that these clusters are formed approximately by the same time. Thus, the gas mass fraction is defined as: $f_{\text {gas }} \equiv M_{\text {gas }} / M_{\text {tot }}$, where $M_{\text {gas }}$ is the X-rays gas mass and $M_{t o t}$ is the dynamical total mass from the galaxy cluster respectively. To relate $f_{\text {gas }}$ with the parameters of a particular cosmological model we can write $M_{\text {gas }}$ and $M_{\text {tot }}$ in terms of $d_{A}(z)$ as follows [26].

$$
f_{\text {gas }}^{\Lambda C D M}(z) \equiv \frac{b}{1+\alpha} \frac{\Omega_{b}}{\Omega_{0 m}}\left(\frac{d_{A}^{\Lambda C D M}(z)}{d_{A}(z)}\right)^{3 / 2},
$$

where $d_{A}(z)$ is the angular diameter distance for a cosmological model and $d_{A}^{\Lambda C D M}(z)$ is the angular diameter distance for a reference model, in this case the standard model $\Lambda C D M . \Omega_{b}$ and $\Omega_{0 m}$ are the baryonic density parameter and the today dark matter density parameter of the Universe respectively. Here $b$ is a bias factor which relates the baryonic fraction from the clusters and the Universe. The constant $\alpha$ relates the baryonic luminous mass and the baryonic gas mass. This constant is $\alpha \approx 0.19 \sqrt{h}[4]$ being $h$ the normalized Hubble constant. Here, we use the $f_{\text {gas }}$ measurements from 42 galaxy clusters obtained by [5]. We use 42 measurements of $f_{\text {gas }}$ of galaxy cluster from [5]. Thus, the $\chi^{2}$ formula to test the different cosmological models we have

$$
\begin{aligned}
& \chi_{f_{\text {gas }}}^{2}\left(z_{i}, \Theta\right)=\sum_{i=1}^{42} \frac{\left[f_{\text {gas }}^{\Lambda C D M}\left(z_{i}, \Theta\right)-f_{\text {gas }}\left(z_{i}, \Theta\right)\right]^{2}}{\sigma_{f_{\text {gas }}}^{2}} \\
& +\left(\frac{\Omega_{b} h^{2}-0.0214}{0.0020}\right)^{2}+\left(\frac{h-0.72}{0.08}\right)^{2}+\left(\frac{b-0.824}{0.089}\right)^{2}(4)
\end{aligned}
$$

where $f_{\text {gas }}$ is observational gas mass fraction data [5], $f_{\text {gas }}^{\Lambda C D M}(z)$ is the gas mass fraction of the cosmological models tested and $\sigma_{f_{\text {gas }}}$ is the error of data. In the former expression we have considered constant values for $b=0.824$ [4].

\subsection{Gravitational lensing}

Strong gravitational lensing occurs whenever the light rays of a source are strongly deflected by the lens that multiples images can be produced. The position of these images depend on the properties of the lens mass distribution [24]. Because the Einstein radii, $\theta_{E}$, also depends on a cosmological model, the SL observations can be used as an additional method to probe the nature of dark matter and dark energy [10]. Another important lensing observable that can be used to infer cosmological parameters is the size of the Einstein radii. In this paper, we use the method which consists in comparing the ratio $\mathcal{D}$ of angular diameter distances between lens and source, $d_{A}\left(z_{l}, z_{s}\right)$, and between observer and lens, $d_{A}\left(0, z_{s}\right)$, with its observable counterpart $\mathcal{D}^{o b s}$ given by

$$
\begin{aligned}
& \mathcal{D}\left(z_{l}, z_{s}\right)=\frac{d_{A}\left(z_{l}, z_{s}\right)}{d_{A}\left(0, z_{s}\right)}=\frac{\int_{z_{l}}^{z_{s}} d z^{\prime} / E\left(z^{\prime}, \Theta\right)}{\int_{0}^{z_{s}} d z^{\prime} / E\left(z^{\prime}, \Theta\right)}, \\
& \mathcal{D}^{o b s}=\frac{c^{2} \theta_{E}}{4 \pi \sigma_{S I S}^{2}},
\end{aligned}
$$

where $\sigma_{S I S}$ is the SIS velocity dispersion and $E(z, \Theta) \equiv$ $H(z, \Theta) / H_{0}$, being $H(z, \Theta)$ the Hubble parameter of the cosmological model to be tested and $H_{0}=H(0, \Theta)$. $\Theta$ is the vector of the parameters of the cosmological model [16]. Here, we use a sample of 80 strong lensing systems by [10] which contains 70 data points from SLACS and LSD and 10 data points from galaxy clusters. The fit of the theoretical models to strong lensing observations can be found by the minimization of

$$
\chi_{S L}^{2}=\sum_{i=1}^{80} \frac{\left(\mathcal{D}_{i}^{o b s}-\mathcal{D}_{i}^{t h}\right)^{2}}{\sigma_{\mathcal{D}, i}^{2}}
$$

where the sum is over the sample and $\sigma_{\mathcal{D}, i}^{2}$ denotes the variance of $\mathcal{D}_{i}^{o b s}$. Additionally with these data sets and in order 
to diminish the degeneracy between the free parameter of the models, we use 580 Supernovae data (SNIa) from Union 2.1 [34], CMB (Planck13) [1], as well as data from BAO (BOSS, WiggleZ, SDSS, 6dFGS) observations, adopting the three measurements of $A(z)$ obtained in [7, 11], and using the covariance among these data given in [31]. (See appendix 6).

\section{Theoretical framework and dark energy models}

The concordance model $\Lambda C D M$ is the most accepted today, which predicts that the universe consists of approximately $4 \%$ of luminous matter, 26\% of Cold Dark Matter (CDM) and about $70 \%$ is a exotic component known as dark energy (DE) which it is traditionally presented as the main responsible for the late accelerated expansion of the Universe. In $\Lambda \mathrm{CDM}$ model, the CDM is made up of colisionless non baryonic particles and the DE is provided by the cosmological constant $\Lambda$ with an equation of state $(\operatorname{EoS}) w=-1$. This model is in excellent agreement with the cosmological observations of the anisotropies of the $\mathrm{CMB}$ radiation, baryonic acoustic oscillations (BAO) and Supernova Ia (SNIa). Nevertheless, the standard model has some fundamental problems related to the nature of the dark matter and dark energy [21, 2]. In the context of DE, there are several theoretical arguments against a cosmological constant. One is the coincidence problem, that is, why today the value of dark energy density is of the same order of magnitude than dark matter density. Other important fundamental issue is the fine tuning of the present value of $\Lambda$ which is completely in disagreement with the predictions of particle physics [36, 18]. Therefore, several dark energy models with a dynamical EoS have been proposed to alleviate the problems of the cosmological constant [21].

In order to put constraints on DE cosmological models using $d_{A, \text { cluster }}, f_{\text {gas }}$ and SGL from galaxy clusters we need to calculate the theoretical angular diameter distance of the model and then compare it with the observations. In addition, to investigate whether a cosmological model can predict an accelerated expansion phase of the Universe, we must study the behavior of the deceleration parameter $q(z)$. The angular diameter distance for a Friedmann-Lemaitre-Robertson-Walker model universe, for a source at redshift $z$ is

$$
d_{A}(z, \Theta)=\frac{3000 h^{-1}}{(1+z)} \frac{1}{\sqrt{\left|\Omega_{k}\right|}} \sin \varsigma\left(\int_{0}^{z} \frac{\sqrt{\left|\Omega_{k}\right|}}{E(z, \Theta)} d z\right),
$$

where $h=H_{0} / 100 \mathrm{kms}^{-1} \mathrm{Mpc}^{-1}$ is dimensionless Hubble parameter and the function $\sin \varsigma(x)$ is defined such that it can be $\sinh (x)$ when $\Omega_{k}>0, \sin (x)$ when $\Omega_{k}<0$ and $x$ when $\Omega_{k}=0$ [22]. In the standar FLRW cosmology, the expansion rate as a function of the scale factor $H(a)$ is given by the Friedmann equation as:

$$
E^{2}\left(a, \Omega_{i}\right)=\Omega_{r} a^{-4}+\Omega_{m} a^{-3}+\Omega_{k} a^{-2}+\Omega_{X} e^{3 \int_{a}^{1} \frac{d a^{\prime}}{a^{\prime}}\left(1+w\left(a^{\prime}\right)\right)}(9)
$$

where $H(a) / H_{0}=E\left(a, \Omega_{i}\right), H_{0}$ is the curent value of the expansion rate and the scale factor is related to redshift as $1+z=$ $a^{-1}$. In the equation $90 \Omega_{i}$ is the current energy density divided by the critical density today $\rho_{c r i}=3 H_{0}^{2} / 8 \pi G$, In the form of the $i$-th component of the fluid density of: radiation $\left(\Omega_{r}\right)$, matter $\left(\Omega_{m}\right)$, curvature $\left(\Omega_{k}\right)$ and dark energy $\left(\Omega_{X}\right)$. The ratio of the pressure to the energy density $\omega(a)=p(a) / \rho(a)$ is the EoS of dark energy, the which divide our models into two cases: in one the energy density of the fluid is constant and in the other the energy density of the fluid is dynamic. In all cosmological models, the density parameter of curvature is free $\Omega_{k}$ and on which constraints are obtained. For each model of dark energy corresponds a vector of parameters $\Theta_{i}^{\text {model }}=\left\{\theta_{i}, \Omega_{i}\right\}$, where $\theta_{i}=$ $\left\{h, \Omega_{b}\right\}$ for the analysis of the present work. The deceleration parameter $q(z)$ is defined as 29]

$$
q(z)=-\frac{\ddot{a}(z) a(z)}{\dot{a}^{2}(z)}=\frac{1+z}{E(z, \Theta)} \frac{d E(z, \Theta)}{d z},
$$

being $a$ the scale factor of the Universe.

\section{1. $\Lambda C D M$}

We begin our analysis with the standard cosmological model. In this paradigm, the DE is provided by the cosmological constant $\Lambda$, with an EoS, such that $w=-1$. In this model the dimensionless Hubble parameter $E^{2}(z, \Theta)$ is given by

$$
E^{2}(z, \Theta)=\Omega_{r}(1+z)^{4}+\Omega_{m}(1+z)^{3}+\Omega_{k}(1+z)^{2}+\Omega_{X}
$$

where $\Omega_{m}$ and $\Omega_{X}=\Omega_{\Lambda}=1-\Omega_{m}-\Omega_{k}-\Omega_{r}$ are the density parameters for matter and dark energy respectively and $\Omega_{r}$ is radiation parameter. The parameter vector is $\Theta_{i}=\left\{h, \Omega_{b}, \Omega_{m}, \Omega_{k}\right\}$ and the best fit is shown in Table 1

\begin{tabular}{ll}
\hline$\Lambda$ Cold Dark Matter model & \\
\hline \hline$h=0.7063 \pm 0.0067$ & $\Omega_{k}=0.0048 \pm 0.0024$ \\
$\Omega_{m}=0.2839 \pm 0.0046$ & $\Omega_{b}=0.04411 \pm 0.00099$ \\
\hline
\end{tabular}

Table 1: Best fit parameters with all data set to $\Lambda C D M$ model.

\section{2. $\omega C D M$ model}

An extension of the standard model where $\Lambda$ has $w=-1$ is to consider that the EoS still is constant but its value can deviate of -1 . In this case the dimensionless Hubble parameter $E^{2}(z, \Theta)$ for a FLRW universe with curvature reads as

$$
\begin{aligned}
E^{2}(z, \Theta)= & \Omega_{r}(1+z)^{4}+\Omega_{m}(1+z)^{3}+ \\
& \Omega_{k}(1+z)^{2}+\Omega_{X}(1+z)^{3(1+w)},
\end{aligned}
$$

where $\Omega_{X}=\left(1-\Omega_{m}-\Omega_{k}-\Omega_{r}\right) . \quad$ In this model the set of free parameters are $\Theta=\left\{h, \Omega_{b}, \Omega_{k}, \Omega_{m}, \omega\right\}$. As in the case of $\Lambda \mathrm{CDM}$ model, first we estimate the parameters using the data from galaxy clusters. Then, we use all observational data. The best fit values are showed in the Table 2 


\begin{tabular}{ll}
\hline$\omega$ Cold Dark Matter model & \\
\hline \hline$h=0.7080 \pm 0.0070$ & $\Omega_{k}=0.0007 \pm 0.0028$ \\
$\Omega_{m}=0.2839 \pm 0.0049$ & $\Omega_{b}=0.0437 \pm 0.0010$ \\
$\omega=-1.0862 \pm 0.0010$ & \\
\hline
\end{tabular}

Table 2: Best fit parameters with all data set to $\omega C D M$ model.

\subsection{Chevalier-Polarski-Linder model}

Other simple extension to the standard scenario is to allow that equation of state of dark energy varyies with time or redshift via some parametrization. One of the most popular function is the Chevallier-Polarski-Linder (CPL) parametrization [17] [25] given by

$$
w(z)=w_{0}+w_{1} \frac{z}{1+z},
$$

where $w_{0} \mathrm{y} w_{1}$ are constants to be fitted. The dimensionless Hubble parameter $E(z)$ for some parametrization can be expressed as

$$
E^{2}(z)=\Omega_{r}(1+z)^{4}+\Omega_{k}(1+z)^{2}+\Omega_{m}(1+z)^{3}+\Omega_{X} X(z),(14)
$$

where $\Omega_{X}=\left(1-\Omega_{k}-\Omega_{m}-\Omega_{r}\right)$ and

$$
X(z)=(1+z)^{3\left(1+w_{0}+w_{1}\right)} \exp \left[-\frac{3 w_{1} z}{1+z}\right]
$$

The free parameters to be constrained are $\Theta=$ $\left\{h, \Omega_{b}, \Omega_{k}, \Omega_{m}, w_{0}, w_{1}\right\}$. The best fit values using galaxy clusters and all the cosmological tests are showed in the Table 3 .

\begin{tabular}{ll}
\hline Chevalier-Polarski-Linder model & \\
\hline \hline$h=0.7059 \pm 0.0075$ & $\Omega_{k}=-0.0032 \pm 0.0035$ \\
$\Omega_{m}=0.2865 \pm 0.0058$ & $\Omega_{b}=0.0439 \pm 0.0011$ \\
$\omega_{0}=-0.92 \pm 0.15$ & $\omega_{1}=-1.09 \pm 0.90$ \\
\hline
\end{tabular}

Table 3: Best fit parameters with all data set to $C P L$ model.

\subsection{Interacting Dark Energy model}

In interacting dark energy (EDE) models there is a relation between the energy density of dark energy $\rho_{x}$ and the energy density of dark matter $\rho_{m}$ that could alleviate the cosmic coincidence problem. For this the general approach is common to introduce an interacting term in the right side of continuity equations in the following way [6]

$$
\begin{aligned}
\dot{\rho_{m}} & +3 H \rho_{m}=\delta H \rho_{m}, \\
\dot{\rho_{x}} & +3 H\left(1+w_{x}\right) \rho_{x}=-\delta H \rho_{m},
\end{aligned}
$$

where $w_{x}$ is the equation of state of dark energy and $\delta$ is a constant interacting term to be fitted with the observations. Thus, the dimensionless Hubble parameter for this interacting model is as follows

$$
\begin{aligned}
E^{2}(z, \Theta)= & \Omega_{r}(1+z)^{4}+\Omega_{k}(1+z)^{2}+\Omega_{m} \Psi(z) \\
& +\Omega_{X}(1+z)^{3\left(1+w_{x}\right)},
\end{aligned}
$$

where $\Omega_{X}=\left(1-\Omega_{m}-\Omega_{k}-\Omega_{r}\right)$ and

$$
\Psi(z)=\frac{\left(\delta(1+z)^{3\left(1+w_{x}\right)}+3 w_{x}(1+z)^{3-\delta}\right)}{\delta+3 w_{x}}
$$

This model is characterized by six parameters $\Theta=$ $\left\{h, \Omega_{b}, \Omega_{k}, \Omega_{m}, w_{x}, \delta\right\}$. We show the best fit values of these parameters in Table 4

\begin{tabular}{ll}
\hline Interacting Dark Energy model & \\
\hline \hline$h=0.7186 \pm 0.0097$ & $\Omega_{k}=0.0211 \pm 0.0044$ \\
$\Omega_{m}=0.3201 \pm 0.0049$ & $\Omega_{b}=0.0424 \pm 0.0012$ \\
$\omega_{x}=-0.8163 \pm 0.0031$ & $\delta=-0.0188 \pm 0.0030$ \\
\hline
\end{tabular}

Table 4: Best fit parameters with all data set to IDE model.

\subsection{Early Dark Energy model}

In the early dark energy (EDE) scenarios it is proposed that the energy density of dark energy can be significant at high redshifts. This can be if the dark energy tracks the dynamics of the background fluid density [32]. These models could ameliorate the coincedence problem of the cosmological constant. Here, we generalize the EDE model proposed by [20] adding a curvature term. The dimensionless Hubble parameter for this EDE model is

$$
E^{2}(z, \Theta)=\frac{\Omega_{r}(1+z)^{4}+\Omega_{m}(1+z)^{3}+\Omega_{k}(1+z)^{2}}{1-\Omega_{X}},
$$

where $\Omega_{X}$ is given by

$$
\Omega_{X}=\frac{\Omega_{X_{0}}-\Omega_{e}\left[1-(1+z)^{3 w_{0}}\right]}{\Omega_{X_{0}}+f(z)}+\Omega_{e}\left[1-(1+z)^{3 w_{0}}\right]
$$

and

$$
f(z)=\Omega_{m}(1+z)^{-3 w_{0}}+\Omega_{r}(1+z)^{-3 w_{0}+1}+\Omega_{k}(1+z)^{-3 w_{0}-1}(21)
$$

such that $\Omega_{X_{0}}=1-\Omega_{m}-\Omega_{k}-\Omega_{r}$ is the current dark energy density. Thus, we have five free parameters $\Theta=$ $\left\{h, \Omega_{b}, \Omega_{k}, \Omega_{m}, \Omega_{e}, \omega_{0}\right\}$. The best fit is shown in Table 5 .

\begin{tabular}{ll}
\hline Early Dark Energy model & \\
\hline \hline$h=0.714 \pm 0.011$ & $\Omega_{k}=0.0138 \pm 0.0041$ \\
$\Omega_{m}=0.3241 \pm 0.0053$ & $\Omega_{b}=0.0421 \pm 0.0012$ \\
$\Omega_{e}=0.055 \pm 0.015$ & $\omega_{0}=-1.151 \pm 0.054$ \\
\hline
\end{tabular}

Table 5: Best fit parameters with all data set to $E D E$ model. 

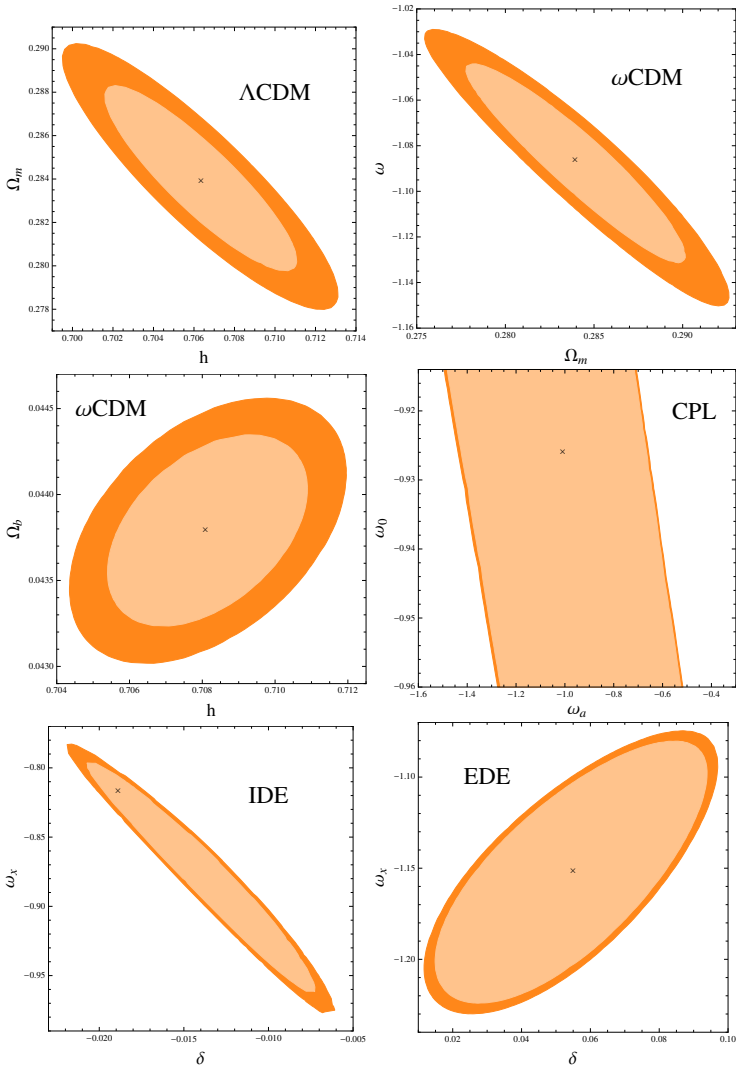

Figure 1: Diagrams of statistical confidence marginalizing different cosmological parameters at $1 \sigma, 2 \sigma$ and $3 \sigma$ for the cosmological models.

\section{Analysis and results}

The maximum likelihood estimate for the best fit parameters is:

$$
\mathcal{L}_{\text {max }}=\exp \left[-\frac{1}{2} \chi_{\text {min }}^{2}\right]
$$

If $\mathcal{L}_{\max }$ has a Gaussian errors distribution, then $\chi_{\min }^{2}=$ $-2 \ln \mathcal{L}_{\max }$, So, for our analysis:

$$
\chi_{\text {min }}^{2}=\chi_{S N I a}^{2}+\chi_{C M B}^{2}+\chi_{B A O}^{2}+\chi_{d_{A, \text { cluster }}}^{2}+\chi_{f_{g a s}}^{2}+\chi_{S G L}^{2}
$$

The Figure 1 we shows the diagrams of statistical confidence at $1 \sigma, 2 \sigma$ and $3 \sigma$ for different cosmological models and several parameter space, from a joint analysis. We use the Fisher matrix method to propagate the errors and thus to obtain the associated uncertainties. This method is widely used in the analysis of the constraint of cosmological parameters from different observational data set [3].

In this paper we use the Akaike and Bayesian information criterion (AIC, BIC), which allow to compare cosmological models with different degrees of freedom, with respect to the observational evidence and the set of parameters [30]. The AIC and BIC can be calculated as:

$$
\begin{aligned}
& A I C=-2 \ln \mathcal{L}_{\text {max }}+2 k, \\
& B I C=-2 \ln \mathcal{L}_{\text {max }}+k \ln N,
\end{aligned}
$$

where $\mathcal{L}_{\text {max }}$ is the maximum likelihood of the model under consideration, $k$ is the number of parameters and $\mathrm{N}$ the number of data points. BIC imposes a strict penalty against extra parameters for any set with $N$ data. It is worthy to note that BIC considers the number $N$ of data points used in the fit. Thus, it imposes a strict penalty against extra parameters for any set of data $\ln N>2$. The prefered model is that which minimizes AIC and BIC. However, the absolute values of them are not of interest, only the relative values between the different models [23].. Therefore the "strength of evidence" can be characterized in the form $\triangle A I C=A I C_{i}-A I C_{\text {min }}$ $\triangle B I C=B I C_{i}-B I C_{\text {min }}$, where the subindex $i$ refers to value of $A I C$ ( $B I C)$ for model $i$ and $A I C_{\text {min }}\left(B I C_{\text {min }}\right)$ is the minimum value of $A I C(B I C)$ among all the models [15]. We give the judgements for both criterion in Tables 6 and 7

\begin{tabular}{cc}
\hline$\triangle A I C$ & Level of Empirical Support For Model $i$ \\
\hline $0-2$ & Substantial \\
$4-7$ & Considerably Less \\
$>10$ & Essentially None \\
\hline
\end{tabular}

Table 6: $\triangle A I C$ criterion.

\begin{tabular}{cc}
\hline$\Delta B I C$ & Evidence Against Model $i$ \\
\hline $0-2$ & Not Worth More Than A Bare Mention \\
$2-6$ & Positive \\
$6-10$ & Strong \\
$>10$ & Very Strong \\
\hline
\end{tabular}

Table 7: $\triangle B I C$ criterion.

Thus, if we have a set of models of dark energy first we should estimate the best fit $\chi^{2}$ and then we can apply the $A I C$ and $B I C$ to identify which model is the prefered one by the observations. Table 8 shows the values of $\mathrm{k}, \chi_{\text {min }}^{2}$, AIC and BIC for the DE models from all cosmological tests, Whose data points are: SNIa (580), CMB (3), BAO (7), $d_{A, \text { clusters }}(25), f_{\text {gas }}$ (42), SGL (80). Notice that the $\omega C D M$ model gives the lowest value of AIC and BIC, therefore is the cosmological model most favored by observational data. The $\triangle A I C$ and $\triangle B I C$ values for the other models are measured with respect to $\omega C D M$ and $\Lambda C D M$ respectively. Following [31], we can classify the DE models in two groups: 1 . The models that shows a substantial level of empirical support and not worth more than a bare mention of evidence against to $\omega C D M$ and $\Lambda C D M ; 2$. Essentially none substantial level of empirical support and very strong evidence against to CPL, IDE and EDE models.

\section{History of the expansion}

Figure 2 shows the plot of the deceleration parameter $q(z)$ using only data from galaxy clusters (See Table 9). As expected, the models studied give $q(z)<0$ at late times and $q(z)>0$ at earlier epoch, which means that the history of the expansion is 


\begin{tabular}{lcll}
\hline Model & $\chi_{\min }^{2}$ & Parameters $\left(\Omega_{b}=0.045\right.$ for all models $)$ \\
\hline \hline$\Lambda \mathrm{CDM}$ & 70.38 & $h=0.722 \pm 0.012, \Omega_{m}=0.268 \pm 0.008, \Omega_{k}=-0.13 \pm 0.16$. \\
$\omega \mathrm{CDM}$ & 70.40 & $h=0.722 \pm 0.012, \Omega_{m}=0.268 \pm 0.009, \Omega_{k}=-0.168 \pm 0.884, \omega=-0.973 \pm 0.682$. \\
$\mathrm{CPL}$ & 72.92 & $h=0.720 \pm 0.012, \Omega_{m}=0.275 \pm 0.013, \Omega_{k}=-0.32 \pm 1.35, \omega_{a}=-1.96 \pm 3.85, \omega_{0}=-0.59 \pm 0.46$ \\
$\mathrm{IDE}$ & 73.12 & $h=0.72 \pm 0.10, \Omega_{m}=0.27 \pm 0.11, \Omega_{k}=0.11 \pm 1.58, \omega_{x}=-0.95_{-0.29}^{+0.79}, \delta=4.08_{-6.51}^{+13.80}$. \\
$\mathrm{EDE}$ & 73.09 & $h=0.721 \pm 0.011, \Omega_{m}=0.276 \pm 0.014, \Omega_{k}=0.431, \omega_{0}=-0.98_{-4.51}^{+0.27}, \Omega_{e}=-0.93_{-3.08}^{+1.08}$ \\
\hline
\end{tabular}

Table 9: The best fit values for the free parameters using the only data from galaxy clusters $\left(d_{A, \text { clusters }}+f_{\text {gas }}\right)$.

\begin{tabular}{|c|c|c|c|c|c|c|}
\hline Model & $\mathrm{k}$ & $\chi_{\min }^{2}$ & AIC & $B I C$ & $\triangle A I C$ & $\triangle \triangle B I C$ behavior of dynamical dark energy at low redshift in this mod- \\
\hline$\Lambda C D M$ & 4 & 777.256 & 785.256 & 803.666 & 2.973 & 0.000 els with variation of the density of dark energy over time. \\
\hline$\omega C D M$ & 5 & 772.283 & 782.283 & 805.295 & 0.000 & 1.629 \\
\hline$C P L$ & 6 & 870.757 & 882.757 & 922.372 & 100.474 & 118.706 \\
\hline$I D E$ & 6 & 867.941 & 879.941 & 907.556 & 97.658 & $103.89^{\circ}$ \\
\hline$E D E$ & 6 & 839.966 & 851.966 & 879.581 & 69.683 & the present work we cc \\
\hline
\end{tabular}

Table 8: AIC and BIC analysis to diferent dark energy models using all data sets.

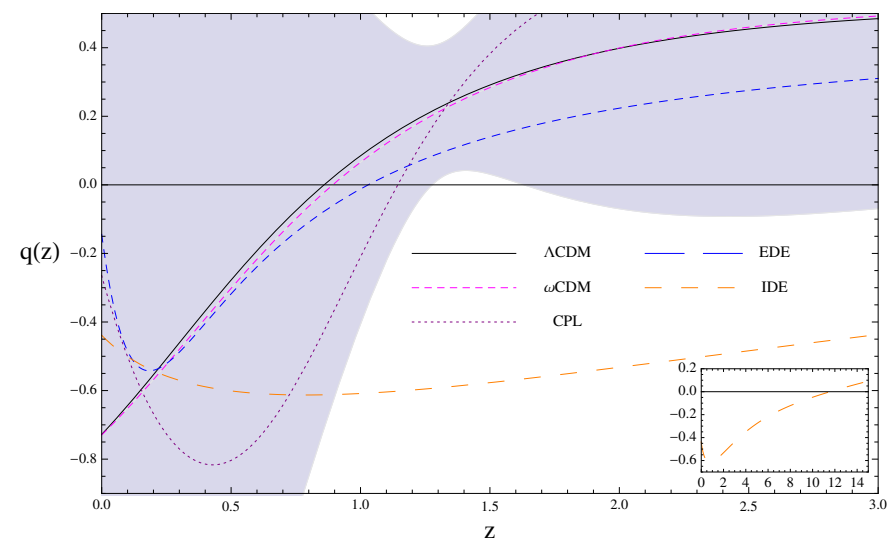

Figure 2: Desaceleration parameter vs redshift using only BAO data, where the gray region corresponds to the best fit to $1 \sigma$ for $\omega C D M$ model, with error propagation only on $\left(d_{A, \text { clusters }}+f_{\text {gas }}\right)$. It is shown the transition deceleratedaccelerated $\left(q\left(z_{t}\right)=0\right)$ and the current value of $\left(q_{0}\right)\left(\Lambda C D M\left(z_{t} \sim 0.85, q_{0}=\right.\right.$ $-0.72), \omega C D M\left(z_{t} \sim 0.88, q_{0}=-0.72\right)$, CPL $\left(z_{t} \sim 1.13, q_{0}=-0.27\right)$, IDE $\left(z_{t} \sim 11.43, q_{0}=-0.44\right)$, EDE $\left.\left(z_{t} \sim 1.1, q_{0}=-0.15\right)\right)$. Note the strange behavior of the deceleration parameter to later times for models of dynamical dark energy (CPL, IDE, EDE).

slowed down in the past and speeded up at present. All cosmological models presents a redshift of transition $\left(z_{t}\right)$ between the two periods (see Figure 2), however, all models of dynamical dark energy present an interesting behavior of slowing down of acceleration at low redshift (late times) using only data from $\mathrm{BAO}$, which can be characterized through the change of sign of the parameter $j(z)$ (CPL: $j\left(z_{\text {low }}\right) \rightarrow 0$, when $z_{\text {low }} \sim 0.42$; IDE: $j\left(z_{\text {low }}\right) \rightarrow 0$, when $z_{\text {low }} \sim 0.83$; EDE: $j\left(z_{\text {low }}\right) \rightarrow 0$, when $\left.z_{\text {low }} \sim 0.16\right)$. We can interpret $\mathrm{j}(\mathrm{z})$ as the slope at each point of $q(z)$, which indicates a change in acceleration (Appendix A.6). This result is consistent with the one presented by J. Barrow, R. Bean and J. Magueijo [8], who raises the possibility of a scenario consistent with the current accelerating universe and does not involve an eternal accelerated expansion. In [12] extensive analysis it is made of this possibility. This can be also a clear models of dark energy, paying particular attention to the data obtained from galaxy clusters and SGL. By using these data sets, we obtained the best-fit parameters for different cosmological models. We use the information criteria including the $\triangle A I C$ and the $\triangle B I C$, to compare different models and to see which is the most favored by current observational data. Our analysis shows that $\omega C D M$ and $\Lambda C D M$ dark energy models is preferred by Akaike and Bayesian criterion. By first time we report that observational data are in favor of the cosmological model $\omega C D M$, at least with AIC. However, the $\Lambda C D M$ model remains the best fit for BIC (See Table 8). In Table 2 and Figure 1 we can see that $\Lambda C D M$ model $(\omega=-1)$ is excluded to a level of statistical confidence of $2 \sigma$ and $1 \sigma(\omega=-1.0862 \pm 0.0010)$ combining all observational data for the present analysis and also from all other cosmological models.

By other side we perform the study of the history of the of cosmic expansion through the $\mathrm{H}(\mathrm{z}), \mathrm{q}(\mathrm{z})$ and $\mathrm{j}(\mathrm{z})$ parameters with data from galaxy clusters, using $d_{A \text {,clusters }}+f_{\text {gas }}$. We find new evidence (presented in previous work with BAO [14]), showing peculiar behavior of the deceleration parameter $\mathrm{q}(\mathrm{z})$ in later times $\left(z_{\text {low }}<0.5\right)$, which is appreciated through change of sign of the parameter $j(z)(+\rightarrow-)$ indicating that the universe could decelerate in the near future (Figure 2). This phenomenon raises the possibility that an accelerated expansion does not imply the eternal expansion, even in the presence of dark energy [12]. This anomalous behavior only appears in dynamic models of dark energy, which in principle can be an indication of the need for a scalar field such as quintessence or phantom. However, the Figure 2 shows that $\Lambda$ still can not be ruled out in this analysis, since all models are in the region of error propagation to $1 \sigma$ of $\omega C D M$ and would have to make a deeper analysis, for example by including other observational evidence for better restriction, which will be presented in next paper.

\section{Acknowledgment}

A. Bonilla wishes to acknowledge to the Universidad Distrital F.J.C. and FIZMAKO group for his academic support. To 
Dr. Beatriz Barbuy (Local Organizing Committee) to their support for the presentation of the present work in the event: "12th International Colloquium on Atomic Spectra and Oscillator Strengths for Astrophysical and Laboratory Plasmas".

\section{References}

[1] Ade, P.A.R. et al., Planck 2013 results. XVI. Cosmological parameters, 2013, [arXiv:1303.5076].

[2] Albrecht, A., Bernstein, G., Cahn, R., et al. 2006, arXiv:astro-ph/0609591

[3] Albrecht A., Amendola L., Bernstein G., Clowe D., Eisenstein D., Guzzo L., Hirata C. and Huterer D. et al., Findings of the Joint Dark Energy Mission Figure of Merit Science Working Group,2009, [arXiv:0901.0721]

[4] S. W. Allen, R. W. Schmidt, H. Ebeling, A. C. Fabian and L. van Speybroeck, Constraints on dark energy from Chandra observations of the largest relaxed galaxy clusters, 2004, MNRAS 353, 457, [astro$\mathrm{ph} / 0405340]$.

[5] S. W. Allen, D. A. Rapetti, R. W. Schmidt, H. Ebeling, G. Morris and A. C. Fabian, Improved constraints on dark energy from Chandra X-ray observations of the largest relaxed galaxy clusters, 2007, MNRAS 383, 879 (2008), [arXiv:0706.0033 [astro-ph]].

[6] L. Amendola, Coupled quintessence, 2000, Phys. Rev. D 62, 043511 (2000), [astro-ph/9908023].

[7] Anderson, L., Aubourg, E., Bailey, S., et al. 2012, Mon. Non. Roy. Astron. Soc., 427, 3435

[8] Barrow, J. D., Bean, R., \& Magueijo, J. 2000, Mon. Non. Roy. Astron. Soc., 316, L41

[9] Bartelmann, M.; Steinmetz, M.; Weiss, A.,Arc statistics with realistic cluster potentials. 2: Influence of cluster asymmetry and substructure, 1995, A\&A, 297, 1, [astro-ph/9408082].

[10] Biesiada, M.; Piórkowska, A.; Malec, B., Cosmic equation of state from strong gravitational lensing systems, 2010, MNRAS, 406, 1055 , [arXiv:1105.0946].

[11] Blake, C., Kazin, E. A., Beutler, F., et al. 2011, Mon. Non. Roy. Astron. Soc., 418, 1707

[12] Bolotin, Y. L., Erokhin, D. A., \& Lemets, O. A. 2012, Physics Uspekhi, $55, \mathrm{~A} 02$

[13] Bonamente, M., Joy, M. K., LaRoque, S.J., Carlstrom, J. E., Reese, E. D. \& Dawson, K.S., Determination of the Cosmic Distance Scale from Sunyaev-Zel'dovich Effect and Chandra X-Ray Measurements of Highredshift Galaxy Clusters, 2006, Astrophys. J. 64725 [astro-ph/0512349].

[14] Bonilla Rivera, A., \& García Farieta, J. 2016, arXiv:1605.01984

[15] Burnham K. P. \& Anderson D. R. 2003, Model Selection and Multimodel Inference, Technometrics, 45, 181

[16] Cao, S.; Pan, Y.; Biesiada, M.; Godlowski, W.; Zhu, Z-H, Constraints on cosmological models from strong gravitational lensing systems, 2012, JCAP, 03, 016, [arXiv:1105.6226].

[17] Chevallier M and Polarski D, Accelerating Universes with Scaling Dark Matter, 2001, Int. J. Mod. Phys. D 10, 213, [gr-qc/0009008].

[18] Copeland, E. J., Sami, M., \& Tsujikawa, S., Dynamics of dark energy, 2006, IJMP D 15, 1753, [arXiv:hep-th/0603057].

[19] De Filippis, E., Sereno, M. , Bautz, M. W. and Longo, G., Measuring the Three-dimensional Structure of Galaxy Clusters. I. Application to a Sample of 25 Clusters, 2005, ApJ 625, 108, [astro-ph/0502153].

[20] Doran \& Robbers, Early Dark Energy Cosmologies, 2006, J. Cosmology Astropart. Phys., 6, 26, [astro-ph/0601544].

[21] Frieman, J. A., Turner, M. S., \& Huterer, D. 2008, ARA\&A, 46, 385

[22] Hogg DW., Distance measures in cosmology, 1999, [astro-ph/9905116].

[23] Liddle A. R., How many cosmological parameters?, 2004, MNRAS 351, L49-L53, [astro-ph/0401198v3].

[24] Limousin, M.; Morandi, A.; Sereno, M.; Meneghetti, M.; Ettori, S.; Bartelmann, M.; Verdugo, T., The Three-Dimensional Shapes of Galaxy Clusters, 2012, [arXiv:1210.3067].

[25] Linder E. V., Mapping the Dark Energy Equation of State, 2003, Phys. Rev. Lett., 90, 091301, [astro-ph/0311403].

[26] S. Nesseris and L. Perivolaropoulos, Crossing the Phantom Divide: Theoretical Implications and Observational Status, 2007, JCAP 0701, 018, [astro-ph/0610092].

[27] Perlmutter, S., Aldering, G., Goldhaber, G., et al. 1999, ApJ, 517, 565
[28] Riess, A. G., Filippenko, A. V., Challis, P., et al. 1998, AJ, 116, 1009

[29] Sandage, A., The Change of redshift and Apparent Luminosity of Galaxies due to the Deceleration of Selected Expanding Universes, 1962, ApJ. 136:319-333.

[30] Schwarz, G., Estimating the Dimension of a Model, 1978, The Annals of Statistics, 6, 471.

[31] Shi K., Huang Y. \& Lu T., A comprehensive comparison of cosmological models from latest observational data, 2012, MNRAS, 426, 2452. [arXiv:1207.5875].

[32] Steinhardt P. J., Wang L., Zlatev I., Cosmological tracking solutions, 1999, Phys. Rev. D., 59, 123504, [astro-ph/9812313].

[33] Sunyaev RA, Zel'dovich YB., The Spectrum of Primordial Radiation, its Distortions and their Significance, 1970, CoASP, 2:66-74.

[34] Suzuki, N., Rubin, D., Lidman, C., et al. 2012, ApJ, 746, 85

[35] Sunyaev, R. A. and Zel'dovich, Ia. B., Microwave background radiation as a probe of the contemporary structure and history of the universe, 1980, ARAA, 18, 537.

[36] Weinberg, S., The cosmological constant problem, 1989, RMP 61, 1.

\section{Appendix}

\section{Appendix A. Cosmography}

In this section we introduce of the most popular cosmological tests, which can help us to uncover some signs of the nature of dynamic dark energy.

It is natural to describe the kinematics of the cosmic expansion through the Hubble parameter $H(t)$ an and its dependence on time, i.e. the deceleration parameter $q(t)$. Following to [12] the scale factor $a(t)$ expands into a Taylor series around the current time $\left(t_{0}\right)$ as:

$$
\frac{a(t)}{a\left(t_{0}\right)}=1+\frac{H_{0}}{1 !}\left[t-t_{0}\right]-\frac{q_{0}}{2 !} H_{0}^{2}\left[t-t_{0}\right]^{2}+\frac{j_{0}}{3 !} H_{0}^{3}\left[t-t_{0}\right]^{3}(\mathrm{~A} .1)
$$

where in general we can have a kinematic description of the cosmic expasion through the set of parameters:

$$
H(t) \equiv \frac{1}{a} \frac{d a}{d t} ; q(t) \equiv-\frac{1}{a} \frac{d^{2} a}{d t^{2}} H(t)^{-2} ; j(t) \equiv \frac{1}{a} \frac{d^{3} a}{d t^{3}} H(t)^{-3}
$$

To characterize whether the universe is currently accelerated or decelerated, the history of expansion is fit through deceleration parameter $q(z) \equiv-\ddot{a}(z) / a(z) H(z)^{2}$. If $q(z)>0, \ddot{a}(z)<0$; then the expansion decelerate, as expected due to gravity (i.e. dark matter, baryonic matter, radiation). The discovery that the universe is currently accelerating, already has about one decade and a half old [27] [28]. The simplest explanation for the accelerating universe is the cosmological constant $\Lambda$, however, there is still no compelling theoretical explanation based on physical foreground and not only phenomenological. To take account information about the dynamics of the expansion we to use (9) and A.2, then we obtain 10 , which directly depends on the cosmological model and its matter-energy content. In general, if $\Omega_{X} \neq 0$ is sufficiently large (i.e. $\Omega_{X}>\Omega_{m}$ ), then $q(z)<0$ and $\ddot{a}(z)>0$, which corresponds to an expansion accelerated the universe as shown by observational data at present, which also indicates a cosmological constant different from zero. If the acceleration of the universe is driven by a new fluid, then it is important to identify signs to determine if the energy density of the fluid is constant or dynamic. 

cle distributed under the terms and conditions of the Creative Commons Attribution (CC BY) license http://creativecommons.org/licenses/by/4.0/). 